\title{
Hadronic Entropy Enhancement and Low Density QGP
}

\author{
A. Delfino ${ }^{1}$, J. B. da Silva ${ }^{1}$, M. Malheiro ${ }^{1}$, M. Chiapparini ${ }^{2}$ and M. E. Bracco ${ }^{2}$ \\ ${ }^{1}$ Instituto de Física, Universidade Federal Fluminense, \\ 24210-340, Niterói, RJ, Brazil. \\ ${ }^{2}$ Instituto de Física, Universidade do Estado do Rio de Janeiro, \\ 20550-900, Maracanã, Rio de Janeiro, RJ, Brazil.
}

(October 29, 2018)

\begin{abstract}
Recent studies show that for central collisions the rising of the incident energy from AGS to RHIC decreases the value of the chemical potential in the HadronQGP phase diagram. Thus, the formation of QGP at RHIC energies in central collisions may be expected to occur at very small values of the chemical potential. Using many different relativistic mean-field hadronic models (RMF) at this regime we show that the critical temperature for the Hadron-QGP transition is hadronic model independent. We have traced back the reason for this and conclude that it comes from the fact that the QGP entropy is much larger than the hadronic entropy obtained in all the RMF models. We also find that almost all of these models present a strong entropy enhancement in the hadronic sector coming from the baryonic phase transition to a nucleon-antinucleon plasma. This result is in agreement with the recent data obtained in the STAR collaboration at RHIC where it was found a rich proton-antiproton matter.
\end{abstract}

Typeset using REVTEX 


\section{INTRODUCTION}

The understanding of nuclear matter under extreme conditions is a crucial and indispensable aim of nuclear and stellar physics, especially with much more experimental information to come with RHIC and Alice/LHC accelerators. It is now widely believed that at sufficiently high energy, in heavy-ion collisions, a central hot region is formed [1, 2, 3, 34. This region is commonly associated with the existence of a Quark-Gluon Plasma (QGP). Following many prescriptions, this hot region expands, cools down and freezes out into hadrons, exhibiting a hadron-gas phase transition. In order to observe this transition to the deconfined phase, some observable signatures have been proposed as, for example, electromagnetic radiation [5,6], strangeness enhancement [7], and $\mathrm{J} / \psi$ suppression [8,9]. High-energy heavy-ion collisions data has brought still more excitation about the predictions of QCD on this subject. In fact, based on the results from different experiments done at CERN-SPS, it is believed that a new state of matter has been formed, the quark matter [10].

Recently, phase transition signatures at resonance-rich matter in heavy ion collisions at RHIC energies has been analyzed in central $\mathrm{Au}+\mathrm{Au}$ collisions at $\sqrt{s}=200 \mathrm{~A} \mathrm{GeV}$ [11, 12]. These studies, using a microscopic transport model (UrQMD) calculation, showed the equilibration of hot matter through a $T \times \mu$ phase diagram. Confronting these results with RHIC, SPS and AGS data, it was shown that for central collisions the rising of the incident energy implies a strong evidence for hadronic-QGP phase transition at very small baryon chemical potentials and temperatures lying between 150 to $200 \mathrm{MeV}$.

Some time ago, using the resonance model, Heinz et al. 13, have studied the Hadron-QGP phase transition at this regime. They found a strong enhancement of the entropy from the hadron to QGP phase. When they included all the resonances and mesons, the entropy of the hadron phase increased but a considerable entropy gap still remained between the two phases. In this paper we will study this transition in relativistic mean field (RMF) models, where we 
have not only a repulsive vector interaction but also an attractive scalar interaction, that was not considered in the resonance model study [13]. This scalar interaction, as we will show in this paper, is quite important in order to study this hadron-QGP transition at low net baryon density. At this regime there is no vector field and the pressure is composed only from the nucleon thermal gas and from the scalar field pressures. The abrupt fall in the nucleon mass at high temperature, seen in many of the RMF hadronic models, is due to the abrupt increasing of the scalar field. This effect resembles a phase transition when the system becomes a dilute gas of baryons in a sea of baryon-antibaryon pairs, and it is caracterized by a large enhancement of the entropy [14,15,16]. Thus, our study in RMF models is quite important because the entropy enhancement depends strongly on the scalar interaction presented in these type of models. It is important to stress, that in all these models the entropy enhancement is maximized at zero chemical potential because when we increase the baryon density, the fall of the nucleon mass with the temperature and also the increase of the entropy become smaller [16]. The importance of the entropy production in relativistic heavy ions collisions has also been addressed to analyze the QGP phase transition, where the entropy density jump at the phase boundary for low net baryon density has been pointed out 17.

At the hadronic level, RMF models are able to describe well a number of nuclear phenomena through different conceptions of meson-nucleon and meson-meson couplings. Most of them follows the basic Walecka model [18] in which improvements have been done in order to better fit the finite nuclei data [19,20,21,22]. It is known that hadronic models may present phase transitions at higher temperatures. For example, in reference [14], hot nuclear matter was extensively studied using the Walecka model regarding the finite temperature behavior for zero baryon density (nucleon-antinucleon plasma). The authors explored this model for different scalar-vector coupling constants to conclude that, depending on the values taken by those constants, the Walecka model may or may not generate a phase transition into a nucleonantinucleon plasma. The order (first or second) of this phase transition depends crucially on 
the scalar coupling constant of the model.

The question we pose in this paper is whether the phase transition for the hadronic sector itself, described from hadronic models, may or not have importance to the QGP phase transition. To answer this question we bring to this discussion a representative class of recent RMF hadronic models [19]. We have also considered derivative coupling models [20,21] and different parametrizations of the non-linear Walecka model such as NL1, NL2, NL3 and NLSH models very well known in the literature [22]. Most of these models were successfully employed to calculate nuclear matter bulk properties as well as the spectra of finite nuclei. The aim of our work is to present a systematic comparative study of a set of hadronic models, at extreme temperatures and very low net baryon density, and the hadronic-QGP phase transition, emphasizing the entropy density behaviour in both phases. The QGP phase is represented by a perturbative QCD derivation [13,23]. We studied the hadronic-QGP phase transition for a broad range of bag constant $B$ and QCD running coupling constant $\alpha_{s}$ values. The particular case of free quarks $\left(\alpha_{s}=0\right)$ is also explicited. This kind of phase transition has been investigated by many authors 24,25]. In particular, aspects of the role played by the nucleon exclude volume have also been studied 26.

As a result, we find that practically all the analyzed hadronic models signalize approximately the same critical QGP temperature. This finding is quite independent whether the hadronic models have or not hadronic phase transition themselves at high temperature. This suggests strongly a RMF hadronic model independence for the QGP phase transition in this regime. To the best of our knowledge, this is a remarkable and unknown feature of the hadronic models. We have traced back the reason for this and conclude that it comes from the fact that the QGP entropy (the slope of the pressure versus temperature) is much larger than the hadronic entropy obtained in all the hadronic models. Therefore, insofar the entropy through sophisticated calorimeters in the future may be a measured quantity, we believe that at very low net baryon density, the enhancement of the entropy may become a signature of a QGP. Previous works 
have already addressed the role played by the entropy at the QGP formation 27,28, 17. We have observed a large jump of the hadronic entropy density, seen in almost all of the RMF models. An enhancement of the entropy per baryon with the rising of the incident energy was also found in recent microscopic transport model (UrQMD) calculations [11,12, However, this model did not include a QGP phase but can be taken as leading credence for our hadronic model phase entropy enhancement signals. This enhancement may favour the hadron-QGP phase transition once it decreases the latent heat for the QGP formation. Thus, we expect in central collisions with a low net baryon density, the formation of a very rich baryon-antibaryon matter in the region of the QGP phase. This results is in agreement with recent data obtained in the STAR collaboration at RHIC where in fact it was found a rich proton-antiproton matter [29].

The outline of this paper is as follows. In Sec. II we present the phase transition calculation. In section III we show our results and discussion, followed by the conclusions in section IV.

\section{THE PHASE TRANSITION CALCULATION AT HIGH T AND $\mu=0$}

At low temperatures and baryon densities (around the nuclear matter ground state), renormalization group arguments [30] show that the QCD running coupling constant is greater than one, indicating confinement of quarks and gluons inside hadrons which are the appropriate degrees of freedom of the nuclear matter in this regime. In this region, QHD based on effective Lagrangians works quite well. The phase transition is supposed to occur at sufficiently high temperatures and densities, where in this case the QCD running coupling constant becomes smaller than one, suggesting deconfinement of quarks and gluons. If one assumes the validity of QHD far beyond the normal nuclear matter ground state, the transition can be characterized by the process of hadrons loosing their identities, and quarks and gluons becoming the elementary degrees of freedom. 
In order to see the hadronic-quark-gluon phase transition we need distinct models for the two different phases of the baryonic matter. We describe the hadronic phase $(\mathrm{H})$ by the models of Ref. 19, 20,21,22 in the Hartree approximation extended to finite temperature at zero net baryon density. For the quark-gluon plasma phase, the equation of state is given given by [13,23. A study of the hadron-QGP phase diagram for finite baryonic density of the models presented in Ref. [19] has been done in Ref. [31]. The Gibbs criteria used in the analysis of the phase transition for zero density is given by $\mu_{H}\left(T_{c}\right)=\mu_{Q G P}\left(T_{c}\right)=0$ and $p_{H}\left(T_{c}\right)=p_{Q G P}\left(T_{c}\right)$.

The functional, used for the hadronic phase, originates from an effective Lagrangian, with additional physical constraints ensuring QCD symmetries [19]. The pressure is

$$
\begin{aligned}
p\left(M^{*}, \rho_{B}\right)= & -\frac{m_{s}^{2}}{g_{s}^{2}} \Phi^{2}\left(\frac{1}{2}+\frac{\kappa_{3}}{3 !} \frac{\Phi}{M}+\frac{\kappa_{4}}{4 !} \frac{\Phi^{2}}{M^{2}}\right) \\
& -\frac{1}{2 g_{v}^{2}}\left(1+\eta_{1} \frac{\Phi}{M}+\frac{\eta_{2}}{2} \frac{\Phi^{2}}{M^{2}}+\frac{\eta_{3}}{3 !} \frac{\Phi^{3}}{M^{3}}\right) m_{v}^{2} W^{2} \\
& -\frac{1}{4 ! g_{v}^{2}}\left(\zeta_{\circ}+\zeta_{1} \frac{\Phi}{M}\right) W^{4}+W \rho_{B} \\
& +\frac{1}{3} \frac{\gamma}{(2 \pi)^{3}} \int d^{3} k \frac{k^{2}}{E^{*}(k)}\left(f_{+}(k, T)+f_{-}(k, T)\right),
\end{aligned}
$$

where $\Phi$ and $W$ are the scalar and vector meson fields respectively, $E^{*}(k)=\left(\mathbf{k}^{2}+M^{* 2}\right)^{1 / 2}$ and $f_{ \pm}(k, T)$ stand for the Fermi-Dirac distribution for baryons (antibaryons). The baryon density $\rho_{B}$ is defined by $\rho_{B}=\frac{\gamma}{(2 \pi)^{3}} \int d^{3} k\left[f_{+}(k, T)-f_{-}(k, T)\right]$. Thus, in the zero net baryon density regime we have the same number of baryons and anti-baryons.

The constants (see Table I) are already adjusted to reproduce some of the bulk properties of the nuclear matter ground state [19]. Here $W 1$ stands for the usual Walecka model. $C 1$ and $Q 1$ are nonlinear $\sigma$ models, having scalar field self-interaction added to the $W 1$ model in order to improve some nuclear matter bulk properties of $W 1$. Most of the developed hadronic models has the $C 1$ structure, given by cubic and quartic scalar field self-interaction . In this aspect it is a very representative model category. The scalar cubic and quartic self-interaction are usually claimed to simulate three-body and four-body forces effects respectively. The others 
as $Q 2, G 1$ and $G 2$ are models representing further improvements by including different scalarscalar, vector-vector and scalar-vector fields couplings. We have also considered for the hadronic phase derivative coupling models [20,21] and different parametrizations of the nonlinear BogutaBodmer model [32, NL1, NL2, NL3 and NLSH models [22].

For the quark-gluon plasma phase, at high temperature, the pressure and the density is given by the expansion [13,23]

$$
\begin{aligned}
p_{Q G P}\left(\mu_{q}, T_{q}\right)= & \frac{8 \pi^{2} T_{q}^{4}}{45}\left(1-\frac{15 \alpha_{s}}{4 \pi}\right)+N_{f}\left[\frac{7 \pi^{2} T_{q}^{4}}{60}\left(1-\frac{50 \alpha_{s}}{21 \pi}\right)\right. \\
& \left.+\left(\frac{\mu_{q}^{2} T_{q}^{2}}{2}+\frac{\mu_{q}^{4}}{4 \pi^{2}}\right)\left(1-\frac{2 \alpha_{s}}{\pi}\right)\right]-B \\
\rho_{Q G P}= & \frac{1}{3} N_{f}\left(\mu_{q} T_{q}^{2}+\frac{\mu_{q}^{3}}{\pi^{2}}\right)\left(1-\frac{2 \alpha_{s}}{\pi}\right),
\end{aligned}
$$

where $B$ is the bag constant, $N_{f}$ is the number of flavors, $\alpha_{s}$ is the QCD running coupling constant, depending on the quark-gluon plasma temperature $T_{q}$ and the quark chemical potential $\mu_{q}$ through the first order perturbative expression

$$
\alpha_{s}=4 \pi\left\{\left(11-\frac{2 N_{f}}{3}\right) \ln \left[\left(0.8 \mu_{q}^{2}+15.622 T_{q}^{2}\right) / \Lambda^{2}\right]\right\}^{-1}
$$

At the zero density regime we are interested we set $\mu_{q}=0$.

The model for the QGP phase which we use in this work has some parameters such as $\Lambda$, $B$ and $N_{f}$. As a first approximation, we examine the simplest case with $N_{f}=2$ (quarks $u$ and $d$ only). The QCD scale parameter $\Lambda$ is fixed at $200 \mathrm{MeV}$, consistent with the current data set. We will use two different bag constants in our analysis: $B^{1 / 4}=174 \mathrm{MeV}$ and $B^{1 / 4}=238$ $\mathrm{MeV}$, corresponding to $B=119 \mathrm{MeV} \mathrm{fm}^{-3}$ and $B=418 \mathrm{MeV} \mathrm{fm}^{-3}$ respectively. These are the limiting values of the broad range of values used in the literature. 


\section{RESULTS AND DISCUSSION}

Figure 1 shows the effective nucleon mass ratio $m^{*} v s$. $T$ for the studied models. We see that ZM, ZM3, C1 and G2 models do not present signals of a first order phase transition in opposite to the others. Then, not all of the hadronic models considered here (twelve in total) have the hot nucleon-antinucleon phase transition in the hadronic sector, manifested by the large decrease of the effective nucleon mass $M^{*}$.

By using the Gibbs criteria of phase equilibrium $p_{1}=p_{2}, \mu_{1}=\mu_{2}=0$ and $T_{1}=T_{2}$ we have obtained the critical temperatures for NL1 ( $188 \mathrm{MeV}), N L 3(191 \mathrm{MeV}), N L S H(192 \mathrm{MeV}$ ), W1 ( $186 \mathrm{MeV}), Q 1(192 \mathrm{MeV}), Q 2(192 \mathrm{MeV})$ and $G 1(191 \mathrm{MeV})$ models. The other models studied in this paper do not present first order hadronic phase transition. The critical temperatures for this hadron nucleon-antinucleon phase transition for hot nuclear matter at the hadronic sector itself are remarkably close to the critical temperature of the hadron-QGP phase transition. This hadron nucleon-antinucleon phase transition where the effective nucleon mass becomes very small can be seen as the Quantum Hadrodynamics version of the chiral phase transition. It is interesting that in recent lattice calculations the chiral and the deconfinement transitions coincide and in our calculation both transitons seem also to appear at the same temperature depending on the value of the bag constant $B$ we choose.

We present in figure 2 the pressure for hadronic-QGP phases (in units of the StefanBoltzmann pressure) as a function of $T$. In this figure we have used the extreme values $B^{1 / 4}=174 \mathrm{MeV}$ and $B^{1 / 4}=238 \mathrm{MeV}$. It is to remark how the QGP critical temperatures are close for all the hadronic models. From this figure we see the critical temperatures very close to $151 \mathrm{MeV}$ for $B^{1 / 4}=174 \mathrm{MeV}$ and around $198 \mathrm{MeV}$ for $B^{1 / 4}=238 \mathrm{MeV}$. The reason lies in the slope for the QGP pressure. This slope is nothing but the entropy density, that we show in figure 3. Notice how far, by a factor around five ( for $\alpha_{s}=0$ ) and around three ( for $\alpha_{s} \neq 0$ ), the entropy amounts are different in both phases. It causes the hadronic model independence 
for the hadronic-QGP critical temperature.

When the hadronic model itself presents the nucleon-antinucleon phase transition it increases substantially the entropy density as can be seen in figure 3 . However, this enhancement is not enough when compared with the QGP entropy. This shows a discontinuity in the entropy signalizing a first order hadron-QGP phase transition, once we are committed with only nucleon-antinucleon in the hadronic phase. Let us also note that the QGP entropy density is independent of the bag constant $B$ since it is given by $\left(\mathcal{E}_{Q G P}+p_{Q G P}\right) / \mathrm{T}$. Note how, in figure 2 for different bag constants, the two slopes are the same for $p_{Q G P}$. However, as pointed out above, the bag constant has effect on the hadronic-QGP value of $T_{c}$. Therefore, if one thinks that the enhancement of the hadronic entropy density would help the QGP formation, only values of $B^{1 / 4}$ greater than $200 \mathrm{MeV}$ should be considered.

We have then included, in an ad hoc fashion, a thermal pion gas contribution to the hadronic phase. By analyzing the behaviour of $\mathcal{E} / p$ as a function of $T$ with and without pions we conclude that the change in the critical phase transition temperature is very small. When we have only nucleons and pions in the hadronic phase, the hadronic-QGP phase transition at very low net baryon density takes place for approximately the same value of $T_{c}$ in all the studied hadronic models. The hadronic-QGP phase transition is essentially given by the QGP phase and lies very close to the temperature where $p_{Q G P}$ crosses zero from below.

The chiral transition version we found in the hadronic models can be identified by a strong decreasing in the effective baryon mass and an abrupt increasing of the entropy density in the hadronic phase ( see Fig. 1 and Fig. 3) . This large hadronic jump of the entropy density favours the hadron-QGP phase transition because it requires a smaller latent heat at the transition compared with the models that do not show that pure hadronic nucleon-antinucleon plasma phase. Thus, from our results, we would expect at very low net baryon density a formation of a very rich baryon-antibaryon matter just before the QGP phase.

It is important to stress that, even with the formation of this rich baryon plasma, the 
entropy enhancement is not enough yet to be compared with the QGP entropy density and still requires a large latent heat at the phase transition. The latent heat and entropy at the transition, as it has been discussed before on the $\mu=0$ central rapidity region [33, 34, 35, may be seen by a large change in the specific volume that slows down the time scale for the conversion of QGP into the hadron matter: a large entropy discontinuity implies a long lifetime of the plasma. This point itself, the entropy discontinuity, has deserved intense work and analysis from many authors [28]. In fact it addresses direct the order of the hadronic-QGP phase transition. We are aware of the limitations of our analysis. The results are dependent on the QGP equation of state we are using, obtained by a perturbative expansion in which we treat the quarks as massless. Note in figure 3 how the entropy density decreases with the increase of the running coupling constant $\alpha_{s}$ that at the phase transition is around 0.4 to 0.6 . This suggests that a non perturbative treatment for the QGP equation of state would reduce the entropy density. Indeed, recent lattice QCD results for the pressure and entropy density 36, 37 have also obtained a strong entropy enhancement close to the phase transition exactly at the order of our critical hadronic entropy density (Figure 3). These lattice results were also reproduced by a non perturbative purely gluonic QCD calculation [38]. Therefore, we should say that the order of the hadron-QGP phase transition is still an open point in our work.

\section{CONCLUSIONS}

Using many different RMF models for the hadronic phase and a perturbative QCD equation of state for the QGP, we study the hadron-QGP phase transition at zero net baryon density and high temperature. We show that the critical temperature $T_{c}$ for this transition is hadronic model independent. We have traced back the reason for this and conclude that it comes from that the QGP entropy (the slope of the pressure versus T) is much larger than the hadronic entropy obtained in all the RMF models. This finding is quite independent whether the hadronic 
models have or not a hadronic phase transition at high temperature when the system becomes a dilute gas of baryons in a sea of baryon-antibaryon pairs.

The hadronic-QGP phase transition at zero net baryonic density takes place for approximately the same value of $T_{c}$ in all the studied hadronic models. Among the studied hadronic models most of them showed a pure hadronic phase transition, the Quantum Hadrodynamics version of the chiral phase transition, seen by a strong decreasing in the effective baryon masses and an abrupt increasing of the entropy density in the hadronic phase. This fact by itself does not change the hadronic-QGP value of $T_{c}$. However, it is important to remark that the latent heat given by $T_{c}\left(S_{Q G P}-S_{H}\right)$ connecting both phases is much lower (but still not small, $S_{Q G P} / S_{H}=3$ with a non zero $\alpha_{s}$, see figure 3) for the hadronic models which present this hadronic phase transition to a baryon-anti-baryon plasma, favouring the Hadron-QGP transition. This result enlarges the knowledge of this transition regarding RMF models and may reinforce different model calculations conjecturing the enhancement of the entropy as a signature for a hadronic-QGP phase transition at low net baryonic density. This signature it is quite important nowadays with the underway experiments, such as the collaboration STAR at RHIC, because recent theoretical studies showed that for central collisions the rising of the incident energy from AGS to RHIC decreases the value of the chemical potential in the Hadron-QGP phase diagram. Thus, the formation of QGP at RHIC energies may be expected to occur at very small values of the chemical potential, exactly the regime we are analyzing in this paper, were we expect a jump on the hadronic entropy density due to the formation of a rich baryon-antibaryon matter. Finally, we stress the importance of the direct measure through calorimeters of this quantity.

Acknowledgements We would like to express our thanks to the Conselho Nacional de Desenvolvimento Científico e Tecnológico (CNPq) for partial financial support. 


\section{REFERENCES}

[1] J. Letessier and J. Rafelski, Int. J. of Mod. Phys.E9, 107 (2000).

[2] D. Zschiesche, S. Bass, M. Bleicher, J. Brachmann, L. Gerland, K. Paech, S. Scherer, S. Soff, C. Spieles, H. Weber, H. Söcker and W. Greiner, nucl-th/0101047 (2001)

[3] Proc. Int. Conf. Quark Matter'99, Nucl. Phys. A661, 1c (1999)

[4] R. Anishetty, P.Koehler, and L. McLerran Phys. Rev. D22, 2793 (1980)

[5] E.V. Shuryak, Phys. Lett. B78, 150 (1978)

[6] K. Kajantic and H. I. Miettinen, Z. Phys. C9, 341 (1981)

[7] J. Rafelski and B. Möller, Phys. Rev. Lett 48, 1066 (1982). ; J. Rafelsky, Phys. Reports 88, $331(1982)$

[8] T. Matsui and H. Satz, Phys. Lett. B178, 416 (1986)

[9] D. Kharzeev and H. Satz, Phys. Lett. B334, 155 (1994)

[10] http://www.cern.ch/CERN/NewState Matter/ with the text of scientific consensus view of the spokesmen of CERN experiments and U. Heinz and M. Jacob, nucl-th/0002042

[11] L. V. Bravina, E. E. Zabrodin, M. Bleicher, S. A. Bass, M. Brandstetter, A. Faessler, C. Fuchst, W. Greiner, M. I. Gorenstein, S. Soff and H. Stöcker, J. Phys. G27 , 421 (2001)

[12] L. V. Bravina, E. E. Zabrodin, S. A. Bass, M. Bleicher, M. Brandstetter, S. Soff, H. Stöcker and W. Greiner, Phys. Rev. C62, 064906 (2000)

[13] U. Heinz, P.R. Subramanian, H. Stöcker and W. Greiner, J. Phys. G12, 1237 (1986).

[14] J.Theis, G.F.Graebner, G.Bucchwald, J.A.Maruhn, W.Greiner, H.Stöcker and J.Polony, Phys. Rev. D28, 2286 (1983). 
[15] R.J. Furnstahl and B.D.Serot, Phys.Rev.C41 , 262 (1990).

[16] M. Malheiro, A. Delfino and C. T. Coelho, Phys. Rev.C58, 426 (1998); J. S. Sa Martins and A. Delfino, Phys. Rev.C61, 044615 (2000).

[17] M. Reiter, A. Dumitru, J. Brachmann,J. A. Maruhn, H. Stöcker and W. Greiner, Nucl. Phys. A643, 99 (1998).

[18] J. D. Walecka, Ann. Phys. 83491 (1974); B. D. Serot and J. D. Walecka, Advances in Nuclear Physics Vol. 16, pp. 1-327 (Plenum, New York, 1986)

[19] R.J.Furnstahl, B.D. Serot and Hua-Bin Tang, Nucl.Phys.A615 (1997) 441.

[20] J. Zimanyi and S. A. Moszkowski, Phys. Rev. C42 (1990) 1416.

[21] A. Delfino, C.T. Coelho and M. Malheiro, Phys. Rev.C51 , 2188 (1995); A. Delfino, C.T. Coelho and M. Malheiro, Phys.Lett.B345, 361 (1995).

[22] P.G. Reinhard, Rep. Prog. Phys. 52, 439 (1989); G. A. Lalazissis, J. Koening and P. Ring, Phys. Rev. c55, 540 (1997); M. M. Sharma, M. A. Nagarajan and P. Ring, Phys. Lett. B312, 377 (1993).

[23] Bo-Qiang Ma, Qi-Ren Zhang, D.H. Rischke and W. Greiner, Phys. Lett. B315,29 (1993).

[24] H. Stöcker and W. Greiner, Phys. Reports 137 (1986) 277 and references there in.

[25] J.C. Collins, and M.J. Perry, Phys. Rev. Lett. 34 (1975) 1353, ; G. Baym and S.A. Chin, Phys. Lett.62B 1976 241, ; B.A. Freedman and L. Mclerran, Phys. Rev.D16, (1977) 1169; J. Kuti, B. Lukacs, J. Polonyi and K. Szlachanvi, Phys. Lett.95B (1980) 75

[26] D.H. Rischke, M.I. Gorenstein, H. Stöcker and W. Greiner, Z. Phys.C51 (1991) 485; O.D. Chernavskaya, Z. Phys.C70 (1996) 517; Bi Pin-zhen and Shi Yao-ming, Z. Phys.C75 (1997) 735. 
[27] J. Letessier, A. Tounsi, U. Heinz, J. Sollfrank and J. Rafelski, Phys. Rev. Lett 70, 3530 (1993).

[28] J. Cleyman, K. Redlich, H. Satz and E. Suhonen, Z. Phys. C58, 347 (1993); A. Leonidov, K. Redlich, H. Satz, E. Suhonen and G. Weber, Phys. Rev. D50, 4657 (1994).

[29] C. Adler et al., Phys. Rev. Lett. 86, 4778 (2001)

[30] E. V. Shuryak, The QCD vacuum, hadrons and the superdense matter, World Scientific Lecture Notes in Physics, Vol. 8.

[31] A. Delfino, M. Chiapparini, M. E. Bracco, L. Castro and S. E. Epsztein, J. Phys. G27, 2251 (2001).

[32] J. Boguta and A. R. Bodmer, Nucl. Phys. A292 414, (1977).

[33] T. Matsui, B. Svetitsky, L.D. McLerran, Phys. Rev. D34, 2047 (1986).

[34] L.D. McLerran and T. Matsui, Proc. Workshop on Experiments for RHIC, BNL,NY, April $15-19(1985)$

[35] B. L. Friman, K. Kajantie, P.V. Ruuskanen, Nucl. Phys. B266, 468 (1986).

[36] F. Karsch, E. Laermann and A. Peikert, Phys. Lett 478, 447 (2000).

[37] F. Karsch, Nucl. Phys. Proc. Suppl. 83, 14 (2000).

[38] J. P. Blaizot, Phys. Rev. Lett 83, 2906 (1999). 


\section{TABLES}

TABLE I. Model parameters, taken from Ref. [3].

\begin{tabular}{ccccccc}
\hline \hline Model & $W 1$ & $C 1$ & $Q 1$ & $Q 2$ & $G 1$ & $G 2$ \\
$m_{s} / M$ & 0.60305 & 0.53874 & 0.53735 & 0.54268 & 0.53963 & 0.55410 \\
$g_{s} / 4 \pi$ & 0.93797 & 0.77756 & 0.81024 & 0.78661 & 0.78532 & 0.83522 \\
$g_{v} / 4 \pi$ & 1.13652 & 0.98486 & 1.02125 & 0.97202 & 0.96512 & 1.01560 \\
$\eta_{1}$ & & 0.29577 & & & 0.07060 & 0.64992 \\
$\eta_{2}$ & & & & -0.96161 & 0.10975 \\
$\kappa_{3}$ & & 1.6698 & 1.6582 & 1.7424 & 2.2067 & 3.2467 \\
$\kappa_{4}$ & & -6.6045 & -8.4836 & -10.090 & 0.63152 \\
$\zeta_{0}$ & & & -1.7750 & 3.5249 & 2.6416 \\
\hline \hline
\end{tabular}




\section{FIGURES}

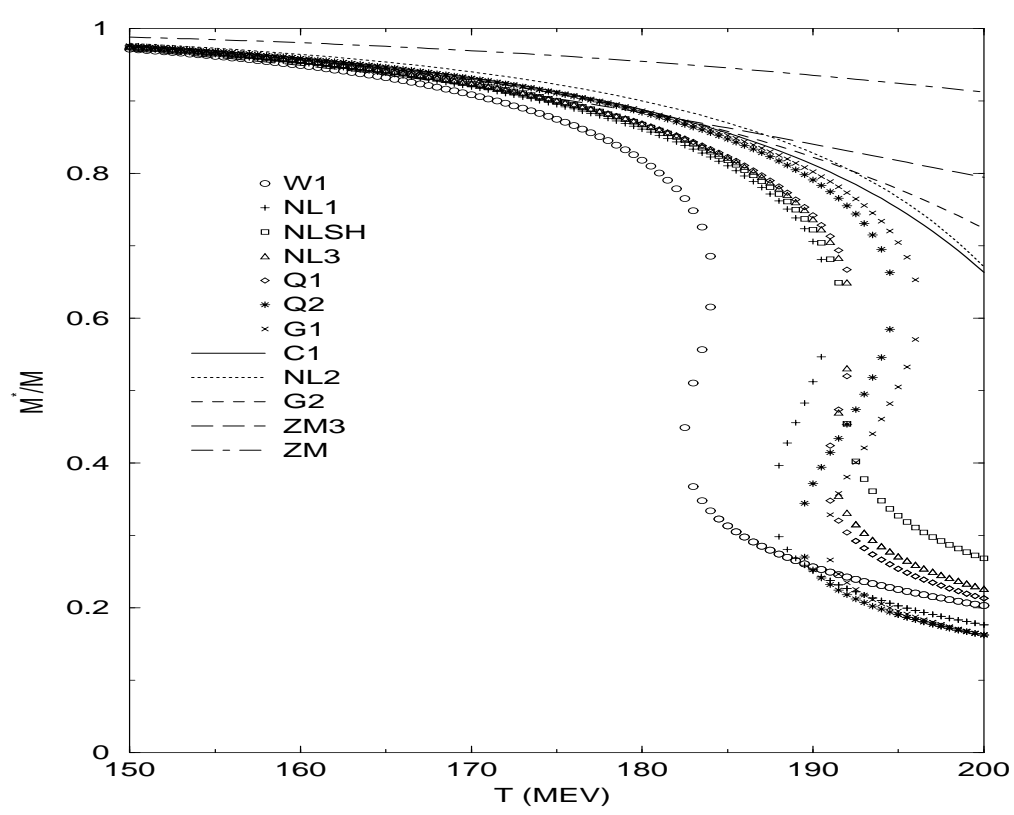

FIG. 1. Effective nucleon mass as a function of the temperature for all the hadronic models.

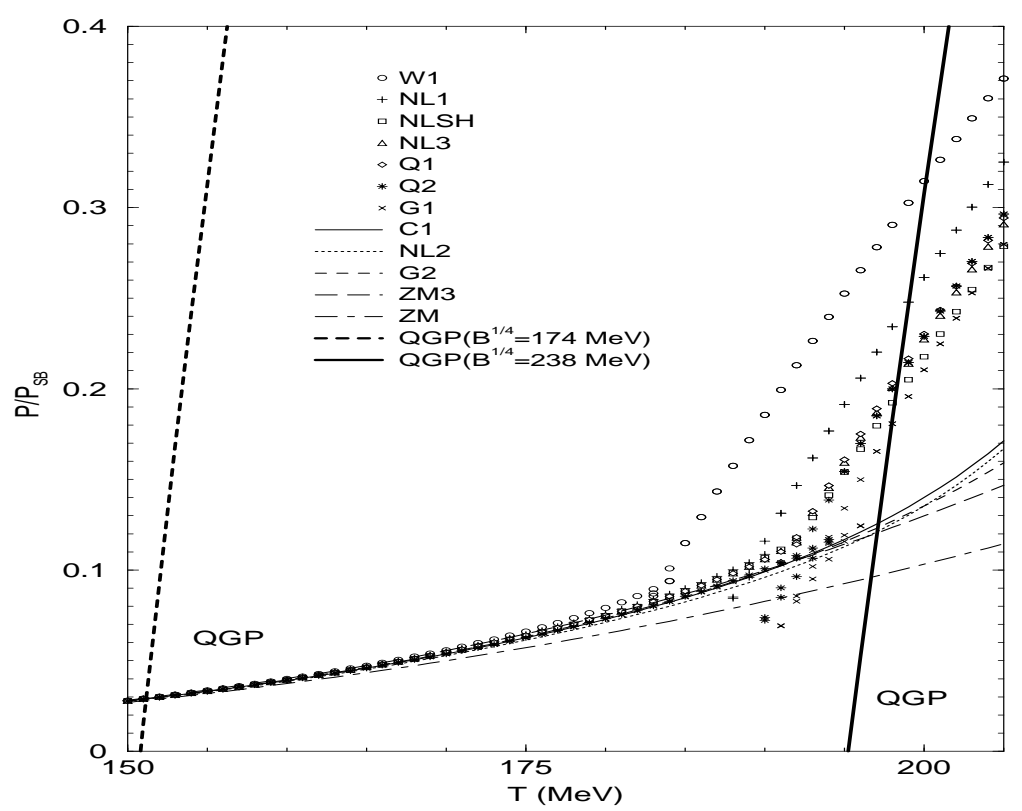

FIG. 2. The pressure for hadronic-QGP phases (in units of the Stefan-Boltzmann pressure) as a function of $T$. 


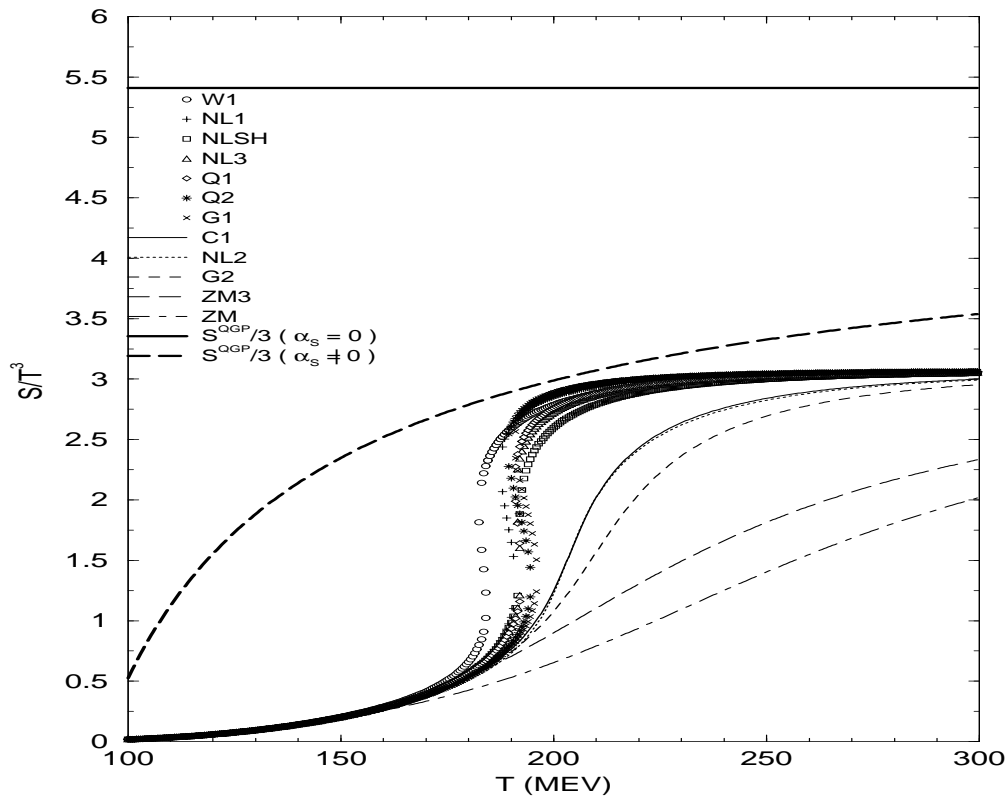

FIG. 3. Entropy density as a function of $T$ for all the hadronic models and for the QGP plasma ( the QGP entropy density is divided by 3 ). 